\title{
Análise de indicadores do serviço de auditoria de um hospital público universitário federal
}

\section{Analysis of indicators of the audit service of a federal university public hospital}

\section{Análisis de indicadores del servicio de auditoría de un hospital público federal universitario}

\author{
Luana de Assis ${ }^{1}$ \\ Lorena Milani Elias Dantas ${ }^{2}$ \\ Suellen Maria Stadler Ribeiro Scolimoski ${ }^{3}$ \\ Bárbara Bowoniuk Wiegand ${ }^{4}$
}

\begin{abstract}
${ }^{1}$ Mestre em Planejamento e Governança Pública pela Universidade Tecnológica Federal do Paraná (Mestrado Profissional). Especialista em Administração Hospitalar pelo Centro Universitário Franciscano do Paraná (Unifae) e especialista em Auditoria em Hospitais, Serviços, Sistemas e Planos de Saúde pela Faculdade Pequeno Príncipe. Administradora do Complexo Hospital de Clínicas da Universidade Federal do Paraná.

E-mail: luana.assis@hc.ufpr.br, Orcid: https://orcid.org/0000-0001-5506-2709

${ }^{2}$ Especialista em Saúde Pública com ênfase em Saúde da Família pelo Centro Universitário Uninter e especialista em Auditoria em Hospitais, Serviços, Sistemas e Planos de Saúde pela Faculdade Pequeno Príncipe. Enfermeira auditora da Unimed

Federação do Estado do Paraná. E-mail: Iorenamilanidantas@yahoo.com.br, Orcid: https://orcid.org/0000-0003-3834-9948

${ }^{3}$ Mestranda em Enfermagem pela Universidade Federal do Paraná (Mestrado Profissional). Especialista em Auditoria em Hospitais, Serviços, Sistemas e Planos de Saúde pela Faculdade Pequeno Príncipe. Enfermeira do Hospital Universitário Cajuru - Grupo Marista. E-mail: suellenstadler@gmail.com, Orcid: https://orcid.org/0000-0002-0987-9504

${ }^{4}$ Mestre em Bioética pela Pontifícia Universidade Católica do Paraná. Advogada. Professora da Faculdade Pequeno Príncipe. E-mail: barbarabowoniuk@hotmail.com, Orcid: https://orcid.org/0000-0003-4459-8086
\end{abstract}


Resumo: Os hospitais públicos devem consolidar uma gestão que trabalhe com os padrões de auditoria do Sistema Único de Saúde (SUS) e que atue com ferramentas estratégicas, entre elas, os indicadores. Esta pesquisa tem como objetivo identificar quais indicadores utilizados na auditoria de hospitais públicos podem ser implantados na auditoria de um hospital público universitário federal do Paraná. Mediante revisão integrativa, com 16 artigos, identificaram-se 43 indicadores utilizados na auditoria de hospitais universitários federais, de ensino e públicos. Após método comparativo e pesquisa documental, constatou-se que, desses 43, 31 (72,09\%) já eram utilizados pela organização estudada. Os demais 12 indicadores ainda não trabalhados foram analisados conforme critérios de implantação e, assim, 10 (83,33\%) foram selecionados para a proposta de implantação no hospital estudado. Ainda, definiram-se também 12 indicadores já trabalhados na instituição paranaense e não mencionados nos hospitais das amostras dos artigos selecionados.

Palavras-chave: indicadores; auditoria; faturamento; hospitais públicos universitários federais; Sistema Único de Saúde.

\begin{abstract}
Public hospitals should consolidate management that works with the Unified Health System (SUS) auditing standards and with strategic tools, among them, the indicators. This research aims to identify which indicators used in the audit of public hospitals can be implemented in the audit of a federal university public hospital in Paraná. Through an integrative review, with 16 articles, we identified 43 indicators used in the audit of federal university hospitals, teaching hospitals, and public hospitals. After comparative method and documentary research, we founded that, of these 43, 31 (72.09\%) were already used by the organization studied. We analyzed the remaining 12 indicators not yet worked according to implantation criteria and, thus, we selected 10 (83.33\%) for the implantation proposal in the studied hospital. Also, we defined 12 indicators already worked in the institution of Paraná and not mentioned in the hospitals of the samples of the selected articles.
\end{abstract}

Keywords: indicators; audit; revenues; federal university public hospitals; Unified Health System.

Resumen: Los hospitales públicos deben consolidar una gestión que funcione con los estándares de auditoría del Sistema Único de Salud (SUS) y que funcione con herramientas estratégicas, entre ellas, los indicadores. Esta investigación tiene como objetivo identificar qué indicadores utilizados en la auditoría de hospitales públicos se pueden implementar en la auditoría de un hospital público universitario federal en Paraná. A través de una revisión integradora, con 16 artículos, se identificaron 43 indicadores utilizados en la auditoría de hospitales universitarios federales, docentes y públicos. Después del método comparativo y la investigación documental, se encontró que, de estos 43, 31 (72.09\%) ya fueron utilizados por la organización estudiada. Los 12 indicadores restantes que aún no funcionan se analizaron de acuerdo con los criterios de implantación y, por lo tanto, se seleccionaron 10 (83,33\%) para la propuesta de implantación en el hospital estudiado. Asimismo, se definieron 12 indicadores que ya funcionaban en la institución de Paraná y no fueran mencionados en los hospitales de las muestras de los artículos seleccionados.

Palabras clave: indicadores; auditoria; facturación; hospitales públicos universitarios federales; Sistema Único de Salud. 


\section{INTRODUÇÃO}

Desde a implantação da Constituição da República Federativa do Brasil, em 1988, e das normativas que fortaleceram as bases do Sistema Único de Saúde (SUS), reconhece-se a necessidade de existir um sistema de auditoria para monitorar a aplicação dos recursos repassados aos prestadores, bem como identificar falhas (contábeis, financeiras, orçamentárias e operacionais) nesse processo.

O Sistema Nacional de Auditoria (SNA/SUS), implementado em 1993, é composto pelos auditores das três esferas (federal, estadual e municipal). Tem como uma de suas principais atribuições verificar se os dados processados pelas organizações prestadoras do SUS, nos sistemas operacionais oficiais, estão em conformidade com os padrões de auditoria e com a legislação. Caso sejam identificadas irregularidades, pode-se bloquear ou ainda solicitar a devolução do ressarcimento fornecido às instituições, realizando-se, assim, o controle dos recursos públicos empregados nas políticas públicas de saúde.

Os hospitais públicos, entidades que trabalham mediante as normativas da Administração Pública, devem estar em conformidade com os requisitos de transparência, economicidade, moralidade e legalidade. Assim, devem consolidar uma gestão que trabalhe com os padrões de auditoria e que atue com ferramentas estratégicas que propiciem melhores resultados, entre elas, os indicadores.

Esta pesquisa tem como objetivo identificar, por meio de revisão integrativa, quais indicadores são utilizados na auditoria de hospitais públicos e apresentar uma proposta para a auditoria de uma unidade gerencial do Complexo Hospital de Clínicas da Universidade Federal do Paraná (CHC/ UFPR). O projeto foi analisado e autorizado pela Gerência de Ensino e Pesquisa da instituição em 22 de maio de 2019.

O propósito é aprimorar o sistema de indicadores que a unidade/ setor já utiliza, de maneira a trazer melhorias como a redução de não conformidades (NCs) em documentos de registro da assistência ambulatorial/ hospitalar; o bloqueio de inconsistências e glosas; e o melhor ressarcimento à instituição. 
A administração financeira sólida propicia recursos e estrutura para o atendimento da população usuária do SUS, que representa cerca de 75\% dos brasileiros. O aperfeiçoamento da gestão dos hospitais públicos universitários federais é de extrema importância ao país, visto que são instituições de referência à assistência de média e alta complexidade na região em que atuam.

Além disso, há o objetivo de verificar quais indicadores já são trabaIhados na instituição paranaense e que não são citados nas bases de dados consultadas. Tem-se, assim, um benefício para a Academia, visto que o material disponível sobre o tema é ainda restrito.

\section{MÉTODO}

A presente pesquisa é classificada quanto ao método como bibliográfica, pois se embasa em materiais publicados, com escopo da revisão de literatura. Dentro dessa abordagem, realizou-se a revisão integrativa, que permite reunir uma diversidade de conhecimentos e estudos sobre o tema. Apresenta-se como um método muito utilizado na área de saúde, devido a sua característica de integrar a teoria com a prática (BOTELHO; CUNHA; MACEDO, 2011).

A revisão integrativa é uma das metodologias mais utilizadas devido a sua organização em etapas e critérios, que consiste desde a definição da questão norteadora até a apresentação dos dados, categorizados e analisados mediante metodologias relacionadas ao assunto abordado. Substitui outras formas sem esse tipo de sustentação, como a narrativa (SOUZA; SILVA; CARVALHO, 2010).

A busca dos artigos foi realizada no período de maio a junho de 2019, nos seguintes portais de dados: Biblioteca Virtual em Saúde Brasil, Saúde Baseada em Evidências e Coordenação de Aperfeiçoamento de Pessoal de Nível Superior (Capes), os quais contêm artigos de diversos bancos de dados, como Lilacs, Medline, SciELO, Base de Dados em Enfermagem, Cadernos Saúde Coletiva, entre outros. Os documentos utilizados foram principalmente artigos e algumas dissertações, pela aproximação com o tema de pesquisa proposto. 
A princípio, seriam usados somente os descritores relacionados a hospitais públicos universitários federais, característica da organização estudada. O CHC/UFPR está inserido no universo dos 50 hospitais universitários federais do país. Porém, com essa delimitação, obtiveram-se apenas quatro artigos, com restrição do material a ser trabalhado. Assim, a decisão foi ampliar a busca com os demais descritores (universitários/de ensino e públicos), a fim de obter um número considerável de informações para se realizar a análise.

De julho a outubro de 2019, executou-se a comparação entre os indicadores obtidos na literatura com os já trabalhados no lócus da pesquisa, a Unidade de Processamento da Informação Assistencial/Setor de Regulação e Avaliação em Saúde (Upia/SRAS). Assim, a pesquisa também é classificada como documental. Realizou-se a análise de planilhas, controles internos e relatórios referentes ao gerenciamento de indicadores de faturamento e da contratualização com o gestor municipal, a Secretaria Municipal de Saúde (SMS).

Primeiramente, definiram-se aqueles indicadores que já eram trabalhados na organização estudada. Após, aqueles não utilizados pela unidade/setor e possíveis de serem implantados, adequando-se à realidade da instituição e considerando os critérios definidos na literatura (GRIFFIN, 2007; OLIVEIRA, 2009; MONTANA; CHARNOV, 2010; ROBBINS; DECENZO, 2012): a) mensurável; b) relacionado a processos estratégicos e críticos; $c$ ) tempo oportuno de mensuração; d) econômico; e) preciso; f) flexível; g) compreensível; h) periódico e i) alinhado a um sistema de múltiplos indicadores.

A elaboração da proposta foi realizada junto da chefia do SRAS (Setor em que a Upia está alocada), de maneira a incluir os indicadores estratégicos aos processos da unidade e do setor. Dos 12 indicadores analisados, 10 foram inclusos na proposta com objetivo de aprimorar o gerenciamento estratégico já realizado.

E, como terceiro resultado, identificaram-se também os 12 indicadores que eram trabalhados no lócus da pesquisa, mas ainda não estavam definidos na literatura. Esse foi um importante marco do estudo, já que se observa que o material publicado sobre o assunto ainda é limitado. 


\section{RESULTADOS}

Após a realização da revisão integrativa nas bases de dados mencionadas, selecionaram-se 16 artigos que se referiam sobre o tema. Os resultados foram organizados conforme o quadro abaixo:

Quadro 1 - Artigos obtidos nas bases de dados por meio da revisão integrativa.

\begin{tabular}{|c|c|c|c|c|}
\hline Data & $\begin{array}{c}\text { Banco } \\
\text { de dados }\end{array}$ & Título & Autores & Periódico \\
\hline $\begin{array}{c}1 . \\
24 / 05 / 2019\end{array}$ & $\begin{array}{c}\text { Bdenf-- } \\
\text { Enfermagem }\end{array}$ & $\begin{array}{l}\text { Os registros de en- } \\
\text { fermagem como } \\
\text { indicadores da quali- } \\
\text { dade do cuidado: um } \\
\text { estudo documental, } \\
\text { descritivo-exploratório } \\
\text { e retrospectivo }\end{array}$ & $\begin{array}{l}\text { VITURI, Dagmar } \\
\text { W.; MATSUDA, } \\
\text { Laura M. }\end{array}$ & $\begin{array}{l}\text { Revista Online } \\
\text { Brazilian } \\
\text { Journal } \\
\text { Nursing, } 2008\end{array}$ \\
\hline $\begin{array}{c}2 . \\
24 / 05 / 2019\end{array}$ & $\begin{array}{c}\text { Bdenf - } \\
\text { Enfermagem }\end{array}$ & $\begin{array}{c}\text { Análise dos indicadores } \\
\text { da qualidade na elabo- } \\
\text { ração da prescrição de } \\
\text { enfermagem em uma } \\
\text { unidade de internação } \\
\text { de um hospital univer- } \\
\text { sitário público }\end{array}$ & $\begin{array}{c}\text { SENTONE, } \\
\text { Andreza D. D. }\end{array}$ & $\begin{array}{l}\text { Biblioteca } \\
\text { Digital } \\
\text { Escola de } \\
\text { Enfermagem } \\
\text { de Ribeirão } \\
\text { Preto, } 2005\end{array}$ \\
\hline $\begin{array}{c}3 . \\
24 / 05 / 2019\end{array}$ & $\begin{array}{c}\text { Bdenf- } \\
\text { Enfermagem }\end{array}$ & $\begin{array}{l}\text { Dez anos buscando a } \\
\text { melhoria da qualidade } \\
\text { do cuidado de enfer- } \\
\text { magem em um hospi- } \\
\text { tal universitário }\end{array}$ & $\begin{array}{l}\text { SILVA, Larissa G. } \\
\text { C. et al. }\end{array}$ & $\begin{array}{l}\text { Revista de } \\
\text { Enfermagem } \\
\text { e Atenção à } \\
\text { Saúde } 2012\end{array}$ \\
\hline $\begin{array}{c}4 . \\
24 / 05 / 2019\end{array}$ & Lilacs & $\begin{array}{c}\text { Auditoria de prescri- } \\
\text { ções de enfermagem } \\
\text { de um hospital de en- } \\
\text { sino paulista: avaliação } \\
\text { da qualidade da assis- } \\
\text { tência } \\
\end{array}$ & $\begin{array}{c}\text { GUEDES, Gisele } \\
\text { G.; TREVISAN, } \\
\text { Danilo D.; } \\
\text { STANCATO, Kátia. }\end{array}$ & $\begin{array}{c}\text { Revista } \\
\text { Administração } \\
\text { Saúde, } 2013\end{array}$ \\
\hline $\begin{array}{c}5 . \\
26 / 05 / 2019\end{array}$ & SciELO & $\begin{array}{c}\text { Avaliação da eficiência } \\
\text { hospitalar por meio da } \\
\text { análise envoltória de } \\
\text { dados }\end{array}$ & $\begin{array}{c}\text { SILVA, Marcia Z.; } \\
\text { MORETTI, Bradlei } \\
\text { R.; SCHUSTER, } \\
\text { Herivelton A. }\end{array}$ & $\begin{array}{l}\text { Revista de } \\
\text { Gestão em } \\
\text { Sistemas de } \\
\text { Saúde, } 2016\end{array}$ \\
\hline
\end{tabular}




\begin{tabular}{|c|c|c|c|c|}
\hline Data & $\begin{array}{c}\text { Banco } \\
\text { de dados }\end{array}$ & Título & Autores & Periódico \\
\hline $\begin{array}{c}6 . \\
01 / 06 / 2019\end{array}$ & $\begin{array}{c}\text { Acta } \\
\text { Scientiarum } \\
\text { (Portal da } \\
\text { UEM) }\end{array}$ & $\begin{array}{c}\text { Informações sobre } \\
\text { causas externas em } \\
\text { internações hospita- } \\
\text { lares: conhecimento e } \\
\text { opiniões de médicos } \\
\text { auditores após uma } \\
\text { intervenção }\end{array}$ & $\begin{array}{l}\text { SANTOS, Hellen } \\
\text { G.; ANDRADE, } \\
\text { Selma M.; } \\
\text { BIROLIM, M. M. }\end{array}$ & $\begin{array}{l}\text { Revista Acta } \\
\text { Scientiarum, } \\
2012\end{array}$ \\
\hline $\begin{array}{c}7 . \\
02 / 06 / 19 \text { e } \\
09 / 06 / 19\end{array}$ & $\begin{array}{l}\text { Lilacs e } \\
\text { SciELO }\end{array}$ & $\begin{array}{l}\text { A informação do setor } \\
\text { de faturamento como } \\
\text { suporte à tomada de } \\
\text { decisão: um estudo de } \\
\text { caso no Hospital Uni- } \\
\text { versitário da UFGD }\end{array}$ & $\begin{array}{c}\text { CINTRA, Renato F. } \\
\text { et al. }\end{array}$ & $\begin{array}{c}\text { Revista } \\
\text { Ciência } \\
\text { \& Saúde } \\
\text { Coletiva, } 2013\end{array}$ \\
\hline $\begin{array}{c}8 . \\
02 / 06 / 19 \text { e } \\
16 / 06 / 19\end{array}$ & Lilacs & $\begin{array}{l}\text { Processo de formação } \\
\text { de contas em um hos- } \\
\text { pital de ensino espe- } \\
\text { cializado em cardiolo- } \\
\text { gia e pneumonia }\end{array}$ & $\begin{array}{l}\text { GUERRER, } \\
\text { Gabriela F. } \\
\text { F.; CASTILHO, } \\
\text { Valéria; LIMA, } \\
\text { Antonio F. C. }\end{array}$ & $\begin{array}{c}\text { Revista } \\
\text { Eletrônica de } \\
\text { Enfermagem, } \\
2014\end{array}$ \\
\hline $\begin{array}{c}9 . \\
11 / 06 / 19\end{array}$ & Lilacs-Express & $\begin{array}{l}\text { Faturamento hospita- } \\
\text { lar aplicado ao serviço } \\
\text { de parto: modelo } \\
\text { alternativo ao fee-for- } \\
\text {-service }\end{array}$ & $\begin{array}{l}\text { COSTA, Elenilde } \\
\text { P. S. R.; ARRAIS, } \\
\text { Alessandra R. }\end{array}$ & $\begin{array}{c}\text { Revista Acta } \\
\text { Paulista de } \\
\text { Enfermagem, } \\
2018\end{array}$ \\
\hline $\begin{array}{c}10 \\
11 / 06 / 19 \text { e } \\
15 / 06 / 19\end{array}$ & $\begin{array}{c}\text { Bdenf - } \\
\text { Enfermagem }\end{array}$ & $\begin{array}{l}\text { O enfermeiro e o regis- } \\
\text { tro de enfermagem em } \\
\text { um hospital público de } \\
\text { ensino }\end{array}$ & $\begin{array}{c}\text { PEDROSA, } \\
\text { Karilena K. A.; } \\
\text { SOUZA, Marília F. } \\
\text { G.; MONTEIRO, } \\
\text { Akemi I. }\end{array}$ & $\begin{array}{c}\text { Revista Rene, } \\
2011\end{array}$ \\
\hline $\begin{array}{c}11 . \\
12 / 06 / 19\end{array}$ & Lilacs & $\begin{array}{l}\text { Estudo da auditoria de } \\
\text { contas em um hospital } \\
\text { de ensino }\end{array}$ & $\begin{array}{l}\text { GUERRER, } \\
\text { Gabriela F. F.; } \\
\text { LIMA, Antonio } \\
\text { F. C.; CASTILHO, } \\
\text { Valéria }\end{array}$ & $\begin{array}{c}\text { Revista } \\
\text { Brasileira de } \\
\text { Enfermagem, } \\
2015\end{array}$ \\
\hline $\begin{array}{c}12 \\
12 / 06 / 19 \text { e } \\
16 / 06 / 19\end{array}$ & $\begin{array}{c}\text { Bdenf - } \\
\text { Enfermagem }\end{array}$ & $\begin{array}{l}\text { Auditoria em regis- } \\
\text { tros de enfermagem: } \\
\text { revisão integrativa da } \\
\text { literatura }\end{array}$ & $\begin{array}{l}\text { CLAUDINO, } \\
\text { Hellen G. et al. }\end{array}$ & $\begin{array}{c}\text { Revista } \\
\text { Enfermagem, } \\
2013\end{array}$ \\
\hline $\begin{array}{c}13 . \\
13 / 06 / 19\end{array}$ & $\begin{array}{c}\text { Bdenf - } \\
\text { Enfermagem }\end{array}$ & $\begin{array}{c}\text { A importância dos } \\
\text { registros de enferma- } \\
\text { gem no faturamento } \\
\text { hospitalar }\end{array}$ & $\begin{array}{c}\text { PERTILLE; } \\
\text { Fabiane; ASCARI; } \\
\text { Rosana A.; } \\
\text { OLIVEIRA, Maíra } \\
\text { C. B. }\end{array}$ & $\begin{array}{l}\text { Revista de } \\
\text { Enfermagem } \\
\text { UFPE On Line, } \\
2018\end{array}$ \\
\hline
\end{tabular}




\begin{tabular}{|c|c|c|c|c|}
\hline Data & $\begin{array}{c}\text { Banco } \\
\text { de dados }\end{array}$ & Título & Autores & Periódico \\
\hline $\begin{array}{c}14 . \\
15 / 06 / 2019\end{array}$ & SciELO & $\begin{array}{c}\text { Avaliação de desempe- } \\
\text { nho econômico-finan- } \\
\text { ceiro dos serviços de } \\
\text { saúde: os reflexos das } \\
\text { políticas operacionais } \\
\text { no setor hospitalar }\end{array}$ & $\begin{array}{l}\text { BONACIM, Carlos } \\
\text { A. G.; ARAUJO, } \\
\text { Adriana M. P. }\end{array}$ & $\begin{array}{c}\text { Revista } \\
\text { Ciência } \\
\text { \& Saúde } \\
\text { Coletiva, } 2011\end{array}$ \\
\hline $\begin{array}{c}15 . \\
16 / 06 / 2019\end{array}$ & $\begin{array}{l}\text { Revista de } \\
\text { Gestão em } \\
\text { Sistemas de } \\
\text { Saúde (RGSS) }\end{array}$ & $\begin{array}{l}\text { Gerenciamento de pro- } \\
\text { cessos: estudo em uma } \\
\text { organização hospitalar } \\
\text { catarinense }\end{array}$ & $\begin{array}{l}\text { DORNELLES, } \\
\text { Treice S.; } \\
\text { GASPARETTO, } \\
\text { Valdirene }\end{array}$ & $\begin{array}{l}\text { Revista de } \\
\text { Gestão em } \\
\text { Sistemas de } \\
\text { Saúde, } 2015\end{array}$ \\
\hline $\begin{array}{c}16 . \\
09 / 06 / 19\end{array}$ & SciELO & $\begin{array}{l}\text { Organizações sociais } \\
\text { de saúde: o modelo do } \\
\text { Estado de São Paulo }\end{array}$ & $\begin{array}{l}\text { IBAÑEZ, Nelson } \\
\text { et al. }\end{array}$ & $\begin{array}{c}\text { Revista } \\
\text { Ciência } \\
\text { \& Saúde } \\
\text { Coletiva, } 2001 .\end{array}$ \\
\hline
\end{tabular}

Fonte: Elaborado pelas autoras, conforme pesquisa de campo (2019).

Obtiveram-se 43 indicadores utilizados na auditoria de hospitais (universitários federais, universitários/de ensino e públicos). Na tabela abaixo, demonstram-se quais indicadores são utilizados na organização estudada (Upia/SRAS).

Quadro 2 - Indicadores utilizados em auditoria de hospitais universitários federais, universitários/de ensino e públicos.

\begin{tabular}{|l|c|c|c|c|}
\hline \multicolumn{1}{|c|}{ Indicador } & $\begin{array}{c}\text { Hospital } \\
\text { Universitário } \\
\text { Federal - } \\
\text { Rede Ebserh }\end{array}$ & $\begin{array}{c}\text { Hospital } \\
\text { Universitário }\end{array}$ & $\begin{array}{c}\text { Hospital } \\
\text { Público }\end{array}$ & $\underline{\underline{\text { Upia/ }}}$ \\
SRAS \\
\hline $\begin{array}{c}\text { 1. NC/ausência evolução de } \\
\text { enfermagem }\end{array}$ & $\mathrm{X}$ & $\mathrm{X}$ & Upia \\
\hline $\begin{array}{c}\text { 2. NC/ausência de prescrição de } \\
\text { enfermagem }\end{array}$ & $\mathrm{X}$ & $\mathrm{X}$ & Upia \\
\hline $\begin{array}{c}\text { 3. NC/ausência de checagem em } \\
\text { prescrições (médica, enfermagem) }\end{array}$ & $\mathrm{X}$ & $\mathrm{X}$ & Upia \\
\hline $\begin{array}{c}\text { 4. Ausência de identificação do } \\
\text { profissional }\end{array}$ & $\mathrm{X}$ & $\mathrm{X}$ & Upia \\
\hline $\begin{array}{c}\text { 5. Ausência de identificação do } \\
\text { paciente }\end{array}$ & & & & \\
\hline
\end{tabular}




\begin{tabular}{|c|c|c|c|c|}
\hline Indicador & \begin{tabular}{|c|} 
Hospital \\
Universitário \\
Federal - \\
Rede Ebserh \\
\end{tabular} & $\begin{array}{l}\text { Hospital } \\
\text { Universitário }\end{array}$ & $\begin{array}{l}\text { Hospital } \\
\text { Público }\end{array}$ & $\underline{\underline{\text { Upia/ }}}$ \\
\hline $\begin{array}{l}\text { 6. Número de documentos com } \\
\text { rasura/ilegível }\end{array}$ & & $x$ & $x$ & Upia \\
\hline 7. Número de partos & & & $x$ & SRAS \\
\hline 8. Número de cirurgias ambulatoriais & & & $x$ & SRAS \\
\hline $\begin{array}{l}\text { 9. Número de atendimentos de } \\
\text { emergência }\end{array}$ & & & $x$ & SRAS \\
\hline 10. Número de consultas & & $\mathrm{x}$ & & Upia \\
\hline 11. Número de consultas externas & & & $x$ & SRAS \\
\hline $\begin{array}{l}\text { 12. Número de consultas por } \\
\text { especialidades }\end{array}$ & $x$ & & & SRAS \\
\hline $\begin{array}{l}\text { 13. Número de outros procedimentos } \\
\text { cirúrgicos }\end{array}$ & & & $x$ & Upia \\
\hline $\begin{array}{l}\text { 14. Número de Autorizações de } \\
\text { Internação Hospitalar (AlHs) } \\
\text { faturadas }\end{array}$ & $x$ & & $X$ & Upia \\
\hline 15. $\mathrm{R} \$$ AlHs faturadas & $x$ & & $\mathrm{x}$ & Upia \\
\hline $\begin{array}{l}\text { 16. Número de procedimentos } \\
\text { ambulatoriais (amb.) faturados }\end{array}$ & $x$ & & & Upia \\
\hline $\begin{array}{l}\text { 17. } \mathrm{R} \$ \text { de procedimentos amb. } \\
\text { faturados }\end{array}$ & $x$ & & & Upia \\
\hline $\begin{array}{l}\text { 18. R\$ fatura AlHs/valor total } \\
\text { faturado }\end{array}$ & $x$ & & & \\
\hline $\begin{array}{l}\text { 19. } \mathrm{R} \$ \text { fatura amb./valor total } \\
\text { faturado }\end{array}$ & $x$ & & & \\
\hline $\begin{array}{l}\text { 20. Número de AlHs por } \\
\text { especialidade }\end{array}$ & $x$ & & & \\
\hline 21. $\mathrm{R} \$$ AlHs por especialidade & $x$ & & & \\
\hline $\begin{array}{l}\text { 22. Número de AlHs por procedência } \\
\text { do paciente }\end{array}$ & $x$ & & & \\
\hline $\begin{array}{l}\text { 23. } \mathrm{R} \$ \text { de AlHs por procedência do } \\
\text { paciente }\end{array}$ & $x$ & & & \\
\hline 24. Taxa de ocupação & $x$ & $x$ & & SRAS \\
\hline 25. Taxa de ociosidade & $x$ & & & SRAS \\
\hline 26. Taxa de permanência & $x$ & & & SRAS \\
\hline $\begin{array}{l}\text { 27. Número de procedimentos } \\
\text { médicos }\end{array}$ & $X$ & & & SRAS \\
\hline
\end{tabular}




\begin{tabular}{|c|c|c|c|c|}
\hline Indicador & $\begin{array}{c}\text { Hospital } \\
\text { Universitário } \\
\text { Federal - } \\
\text { Rede Ebserh }\end{array}$ & $\begin{array}{c}\text { Hospital } \\
\text { Universitário }\end{array}$ & $\begin{array}{l}\text { Hospital } \\
\text { Público }\end{array}$ & $\begin{array}{l}\text { Upia/ } \\
\underline{\text { SRAS }}\end{array}$ \\
\hline $\begin{array}{l}\text { 28. Número de procedimentos de } \\
\text { enfermagem }\end{array}$ & & $x$ & & SRAS \\
\hline $\begin{array}{l}\text { 29. Número de dias entre a entrada } \\
\text { da conta no serviço até a } \\
\text { liberação da fatura }\end{array}$ & & $x$ & & Upia \\
\hline $\begin{array}{l}\text { 30. Não conformidades no prontuário } \\
\text { (não especifica) }\end{array}$ & & $x$ & & Upia \\
\hline $\begin{array}{l}\text { 31. R\$ incluso nas contas após } \\
\text { auditoria }\end{array}$ & & $\mathrm{x}$ & & \\
\hline $\begin{array}{l}\text { 32. } R \$ \text { excluído das contas após } \\
\text { auditoria }\end{array}$ & & $x$ & & \\
\hline 33. Itens e valores de glosas & & $x$ & $x$ & Upia \\
\hline 34. Contas não faturadas & & & $x$ & Upia \\
\hline 35. \% de glosas & & & $x$ & Upia \\
\hline $\begin{array}{l}\text { 36. Número de devoluções por erro } \\
\text { na entrega }\end{array}$ & & & $x$ & Upia \\
\hline 37. $\mathrm{R} \$$ não cobrados por erro & & & $x$ & Upia \\
\hline $\begin{array}{l}\text { 38. Número de glosas pela auditoria } \\
\text { concorrente }\end{array}$ & & & $x$ & \\
\hline $\begin{array}{l}\text { 39. Produtividade (quantidade } \\
\text { de contas fechadas/ n. de } \\
\text { funcionários no faturamento }\end{array}$ & & & $\mathrm{x}$ & Upia \\
\hline 40. Custo por procedimento & & & $x$ & \\
\hline 41. Custo médio por pacientes & & $x$ & & SRAS \\
\hline 42. Custo médio de internação & & $\mathrm{x}$ & & \\
\hline 43. Número de internações & & $x$ & & SRAS \\
\hline
\end{tabular}

Legenda: $\square$ Indicadores presentes na Upia/SRAS

$\square$ Indicadores não trabalhados na Upia/SRAS

Fonte: Elaborado pelas autoras, conforme pesquisa de campo (2019).

Pode-se verificar que, dos 43 indicadores, 31 (72,09\%) já são trabalhados pela Upia ou SRAS. Com relação ao escopo inicial, hospital universitário federal, dos 15 indicadores trabalhados no Hospital Universitário da Grande Dourados/Mato Grosso do Sul (CINTRA et al., 2013), 9 (60\%) estão presentes na instituição paranaense. 
Os 12 indicadores ainda não trabalhados (destacados em cinza na tabela acima) foram analisados conforme os critérios de implantação definidos na literatura (GRIFFIN, 2007; OLIVEIRA, 2009; MONTANA; CHARNOV, 2010; ROBBINS; DECENZO, 2012), a fim de verificar quais seriam inclusos na proposta ao CHC/UFPR.

Quadro 3 - Análise da implantação de indicadores conforme critérios definidos em literatura.

\begin{tabular}{|c|c|c|c|c|c|c|c|c|c|}
\hline $\begin{array}{l}\text { INDICADORES COLETA PESQUISA } \\
\underline{\mathbf{x}} \\
\underline{\text { CRITÉRIOS DE IMPLANTAÇÃO }}\end{array}$ & 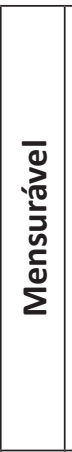 & 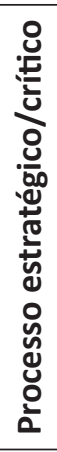 & 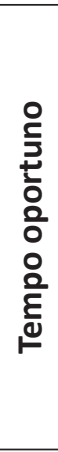 & 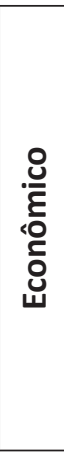 & 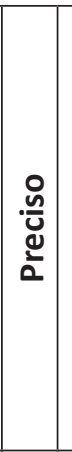 & $\begin{array}{l}\stackrel{\bar{\Phi}}{\bar{x}} \\
\frac{0}{4}\end{array}$ & 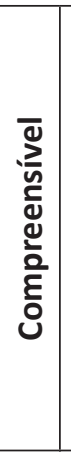 & 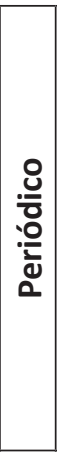 & 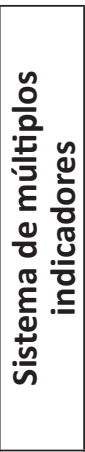 \\
\hline $\begin{array}{l}\text { 2. NC/ausência de prescrição de } \\
\text { enfermagem }\end{array}$ & $x$ & & $x$ & $x$ & $x$ & $x$ & $x$ & $x$ & $x$ \\
\hline 18. R\$ FATURA AlHs/valor total faturado & $x$ & $x$ & $x$ & $x$ & $x$ & $x$ & $x$ & $x$ & $x$ \\
\hline 19. $R \$ \$$ FATURA Amb./valor total faturado & $x$ & $x$ & $x$ & $x$ & $x$ & $x$ & $x$ & $x$ & $x$ \\
\hline 20. Número de AIHS por especialidade & $x$ & $x$ & $x$ & $x$ & $x$ & $x$ & $x$ & $x$ & $x$ \\
\hline $\begin{array}{l}\text { 21. R\$ AlHs por especialidade (substituiu } \\
\text { por } \mathrm{R} \$ \text { AlHs por subgrupo) }\end{array}$ & $x$ & $x$ & $x$ & $x$ & $x$ & & $x$ & $x$ & $x$ \\
\hline $\begin{array}{l}\text { 22. Número de AlHs por procedência do } \\
\text { paciente }\end{array}$ & $x$ & $x$ & $x$ & $x$ & $x$ & $x$ & $X$ & $x$ & $x$ \\
\hline $\begin{array}{l}\text { 23. R\$ de AlHs por procedência do } \\
\text { paciente }\end{array}$ & $x$ & $x$ & $x$ & $x$ & $x$ & & $X$ & $x$ & $x$ \\
\hline $\begin{array}{l}\text { 31. R\$ incluso nas contas após auditoria } \\
\text { (interna) }\end{array}$ & $x$ & $x$ & $x$ & $x$ & $x$ & $x$ & $x$ & $x$ & $x$ \\
\hline $\begin{array}{l}\text { 32. R\$ excluído das contas após auditoria } \\
\text { (interna) }\end{array}$ & $x$ & $x$ & $x$ & $x$ & $x$ & $x$ & $x$ & $x$ & $x$ \\
\hline $\begin{array}{l}\text { 38. Número de glosas pela auditoria } \\
\text { concorrente (externa) }\end{array}$ & $x$ & $x$ & $x$ & $x$ & $x$ & $x$ & $x$ & $x$ & $x$ \\
\hline 40. Custo por procedimento & \multicolumn{9}{|c|}{ NÃO SE APLICA } \\
\hline 42. Custo médio de internação & \multicolumn{9}{|c|}{ NÃO SE APLICA } \\
\hline
\end{tabular}

Fonte: Elaborado pelas autoras, conforme pesquisa de campo (2019). 
O primeiro critério verificado foi aquele relacionado ao "processo estratégico/crítico", aquele que, se tiver falhas não identificadas, causará grandes danos (OLIVEIRA, 2009; ROBBINS; DECENZO, 2012). A análise foi feita junto da chefia do SRAS, responsável pelas unidades e pelo gerenciamento de todos os processos de contratualização com o gestor municipal.

Após, todos os demais critérios foram estudados em conjunto, pelas pesquisadoras. Para a inclusão na proposta de implantação de indicação na Upia/SRAS, definiu-se que seriam aqueles que apresentassem a conformidade com oito ou nove critérios.

Assim, os indicadores selecionados com nove critérios foram:

1. $\mathrm{R} \$$ fatura AlHs/valor total faturado.

2. $R \$$ fatura amb./valor total faturado.

Os dois indicadores serão facilmente obtidos, pois atualmente se têm os indicadores "R\$ fatura AlHs" e "R\$ fatura Amb.". Assim, só será feita a proporção de cada um deles pelo total faturado. Os indicadores são fundamentais para que seja possível a comparação do ressarcimento com custos e gastos para a realização dos procedimentos, de modo a obter o equilíbrio financeiro (IBAÑEZ et al., 2001).

3. Número de AlHs por procedência do paciente.

Também será de fácil obtenção, visto que o sistema interno já tem um relatório que traz essa informação, solicitada anteriormente pela Upia.

4. $\mathrm{R} \$$ incluso nas contas após auditoria (interna).

5. $\mathrm{R} \$$ excluído das contas após auditoria (interna).

Esses dois indicadores poderão ser implantados, tanto para a análise de procedimentos médicos como também de enfermagem, visto que a auditoria da unidade é formada por esses profissionais. O propósito será a identificação de inconsistências que poderiam se tornar glosas e perdas financeiras (PERTILLE; ASCARI; OLIVEIRA, 2018). Essa modalidade de auditoria medirá o volume de recursos que o hospital deixaria de receber em casos de, por exemplo, falta de checagem durante a internação do paciente (DORNELLES; GASPARETTO, 2015).

6. Número de glosas pela auditoria concorrente (externa). 
Este pode ser implantado, porém a sua realização será no momento em que o gestor municipal realizar as auditorias. Ou seja, a periodicidade do levantamento não será de governabilidade do hospital, e sim da SMS. As auditorias poderão ser na modalidade concorrente ou retroativa. No caso de análises retroativas, as diferenças a serem inclusas ou excluídas são pontuadas em rubrica contratual.

A análise e a validação dos dados apresentados pelos prestadores aos auditores da gestão municipal são também a construção das informações de saúde, base para formar políticas que atendam às demandas da sociedade (SANTOS; ANDRADE; BIROLIM, 2012).

O indicador do número de AlHs por especialidade seria possível somente se o Setor de Gestão da Informação e Informática realizasse a inclusão da informação "especialidade" em relatório já existente no sistema interno. A mudança foi apresentada ao setor, bem como as motivações desta pesquisa, e já foi executada em novembro/2019, atingindo todos os critérios de implantação.

Esse indicador traz relacionamentos muito importantes para o processo decisório da gestão, como número de leitos por especialidade versus produção apresentada por cada equipe assistencial. Aquela que tiver mais recursos disponibilizados deve atender com mais efetividade à demanda.

O indicador "R\$ AlHs por especialidade" será substituído por "R\$ AlHs por subgrupo de procedimentos da tabela Sigtap/SUS", já que esta é a informação possível de ser levantada pelo sistema TabWin, em que é disponibilizada toda a produção nacional pelo Ministério da Saúde. Será fundamental para complementar a análise do indicador "número de AlHs por especialidade".

Outro indicador com oito critérios atendidos e que também será incluso na proposta é "NC/ausência de prescrição de enfermagem", mesmo não atendendo isoladamente o critério de "processo estratégico". Entretanto está ligado a um sistema de múltiplos indicadores de não conformidades (NCs) em prontuários de alta hospitalar e a uma série de documentos que são monitorados (descrição de cirurgia, evolução médica, evolução de enfermagem, prescrição médica e enfermagem, resumo de alta, hemoderivados, partograma, entre outros) e que são essenciais para a assistência e gestão 
(SENTONE, 2005; VITURI; MATSUDA, 2008; SILVA et al., 2012; GUEDES; TREVISAN; STANCATO, 2013; COSTA; ARRAIS, 2018).

Há apontamentos definidos como indispensáveis no que tange à evolução de enfermagem, tais como anotação de data e hora, checagem de medicamentos e procedimentos, legibilidade, registro da saída do paciente (alta/óbito) e assinatura do profissional. Se ausentes, podem acarretar danos assistenciais, glosas e processos judiciais (PEDROSA; SOUZA; MONTEIRO, 2011; CLAUDINO et al., 2013; GUERRER; LIMA; CASTILHO, 2015).

$\mathrm{E}$, ainda, o indicador "R\$ de AlHs por procedência do paciente" também com acesso às informações pelo sistema TabWin. Na reunião com a chefia do SRAS, complementou-se esse indicador com "R\$ de perdas de AlHs devido à procedência do paciente". Isso porque todo o prestador tem as regiões de atendimento delimitadas pelo Plano Diretor de Regionalização (PDR). E, caso haja atendimentos de usuários de regionais não inclusas no PDR para determinada instituição, pode-se ter a perda do ressarcimento. Esses indicadores serão complementares e analisados de forma conjunta com o indicador "Número de AlHs por procedência do paciente".

Dos 10 indicadores da proposta, seis são trabalhados em um hospital universitário federal (escopo principal da pesquisa), o Hospital Universitário Federal da Grande Dourados/Mato Grosso do Sul. Os indicadores excluídos devido à não conformidade com a estrutura e divisão de atividades entre a Upia são: "custo por procedimento" e "custo médio de internação". Isso porque outra unidade do setor é responsável por gerenciar essa informação, a Unidade de Contabilidade e Custos. Assim, considerou-se mais adequado manter o foco desses indicadores com essa unidade.

No próximo quadro, apresentam-se os indicadores que são trabaIhados na Upia, mas que não foram identificados na revisão integrativa. Esses podem ser aplicados nas demais organizações hospitalares federais, como também universitárias/de ensino e públicas, para aprimoramento da gestão dos processos de faturamento e da auditoria, bem como redução de perdas financeiras. 
Quadro 4 - Indicadores utilizados na Upia/SRAS e não visualizados na revisão integrativa

\section{SERVIÇO DE FATURAMENTO - UPIa/SRAS}

FATURAMENTO INTERNAÇÃO

1. Tempo de retorno do prontuário ao faturamento após notificação de erro (número de dias)

2. Procedimentos estéticos

3. \% Procedimentos estéticos não faturados versus valor aprovado AlHs

4. Número de órteses e próteses não faturadas - incompatíveis e excedentes

5. Perda de órteses e próteses incompatíveis e excedentes (valor compra item + valor não faturado)

6. \% de órteses e próteses incompatíveis e excedentes versus valor aprovado AlHs

7. Número de medicamentos e exames não faturados - incompatíveis e excedentes

8. Número de cirurgias canceladas sem ressarcimento algum - rejeição 1으 atendimento

9. Perda em cirurgias canceladas sem ressarcimento algum - rejeição 10 atendimento (custo diária + valor não ressarcido)

10. \% de perda em cirurgias canceladas sem ressarcimento algum - 1 o atendimento versus valor aprovado AlHs

\begin{tabular}{|l|}
\hline \multicolumn{1}{|c|}{ FATURAMENTO AMBULATORIAL } \\
\hline 11 . Número de Apacs* criadas \\
\hline 12. Número de Apacs renovadas
\end{tabular}

*Autorizações de Procedimento Ambulatorial de Alto Custo (Apacs).

Fonte: Elaborado pelas autoras, conforme pesquisa de campo (2019).

Nos artigos consultados, houve menção sobre a identificação de erros em contas e prontuários encaminhados ao Serviço de Faturamento (DORNELLES; GASPARETTO, 2015), porém não se apontou o controle do tempo de retorno dos documentos com as correções solicitadas. Esse monitoramento é muito importante, visto que, quanto mais rápido o retorno, em menor tempo possível poderá ser recebido o ressarcimento pela assistência (GUERRER; CASTILHO; LIMA, 2014).

O indicador do número de procedimentos estéticos é relevante, visto que esses não são ressarcidos pelo SUS. Entretanto sua realização é essencial para o atendimento dos requisitos da residência médica em cirurgia plástica, publicados e corroborados na Resolução n. 7, de 8 de abril de 2019. 
Assim, o objetivo do controle da quantidade e do valor desses procedimentos, não faturados pelo SUS, é garantir o equilíbrio dos valores recebidos e custos dispendidos, sendo indispensável para a gestão pública.

Os demais indicadores relacionados ao faturamento de internação têm como objetivo identificar perdas de faturamento e realizar ações para evitar a ocorrência. Na tabela Sigtap/SUS, há compatibilidade de materiais, bem como sua quantidade, que podem ser cobrados em cada procedimento. Porém, em muitas situações, é necessária a utilização de um item não compatível para determinado procedimento, ou ainda em quantidade excedente. Busca-se reduzir essa ocorrência, nos casos em que existe essa possibilidade, desde que a qualidade assistencial ao paciente não seja afetada. O mesmo para medicamentos e exames, que têm a mesma lógica. Esse indicador foi implantado pela Upia em 2019 e está em processo de consolidação de ações.

Outro indicador de perda é quanto às cirurgias canceladas, sem a realização de procedimento algum, e que assim não têm nenhum valor de ressarcimento. Identificam-se os motivos desses cancelamentos pelo resumo de alta e compartilha-se com o Setor Cirúrgico, que realiza o monitoramento, de forma a reduzir as ocorrências.

Esse e demais indicadores permitem a identificação das fragilidades do cenário ideal. Os dados podem ser analisados com as equipes envolvidas de maneira a entender as causas, bem como definir as ações necessárias para bloquear repetições. Esse movimento propicia o aprimoramento contínuo.

E, quanto ao faturamento ambulatorial, os artigos trouxeram indicadores de consultas e demais procedimentos ambulatoriais (BONACIM; ARAUJO, 2011; CINTRA et al. 2013; SILVA; MORETTI; SCHUSTER, 2016), porém sem discriminar a produção mensal de Autorização de Procedimento Ambulatorial de Alto Custo (Apac). É importante esse dado exatamente por ter um maior ressarcimento e, assim, com mais requisitos de atendimento ao gestor municipal. Esses, como todos os demais procedimentos ambulatoriais e de internação, estão contratualizados. Portanto é fundamental monitorar a quantidade realizada para se atingir as metas. 


\section{CONSIDERAÇÕES FINAIS}

Corrobora-se a importância desta pesquisa ao verificar-se que ainda há limitação de estudos sobre um tema de grande relevância para a gestão e a política pública de saúde. O resultado dos 43 indicadores utilizados em auditoria de hospitais públicos e dos 12 indicadores utilizados no hospital universitário federal estudado é uma base que pode nortear os gestores da área, sejam dos prestadores ou dos órgãos de auditoria do SUS.

Ainda, pontua-se a contribuição com o banco de dados sobre os indicadores de auditoria em hospitais públicos, pois o tema é ainda pouco abordado nos cursos de pós-graduação de Auditoria e outros relacionados.

Com relação à organização analisada, Complexo Hospital de Clínicas da Universidade Federal do Paraná, pode-se constatar que há um método de trabalho importante já realizado, visto que 72,09\% dos indicadores obtidos já estão presentes na unidade/setor. Soma-se a esse cenário positivo a existência de condições para a implantação dos 12 indicadores analisados, já que os dois não selecionados para inclusão na Upia poderão ser realizados por outra unidade do setor. Destaca-se que a instituição paranaense está em um processo benéfico da implantação de sua auditoria administrativa da área de faturamento.

O aprimoramento da gestão estratégica desse hospital universitário ou de qualquer outra instituição pública que utilize as informações desta pesquisa atingirá uma melhoria na sua sustentabilidade financeira e, consequentemente, melhores condições de assistência aos cidadãos usuários do SUS.

Como sugestões de estudos futuros, apresenta-se: a) a mesma temática em outros hospitais, públicos e universitários federais; b) outras metodologias complementares à executada para a elaboração da proposta, como avaliações qualitativas para implantação e monitoramento de indicadores; c) e, na organização estudada, análise dos resultados após a implantação dos indicadores propostos, em um período de seis a 12 meses.

\section{AGRADECIMENTO}

As autoras agradecem à Gerência de Ensino e Pesquisa, à Gerência de Atenção em Saúde e ao Setor de Regulação e Avaliação em Saúde do 
Complexo Hospital de Clínicas da Universidade Federal do Paraná (UFPR) pela autorização, contribuição e apoio à realização desta pesquisa.

\section{REFERÊNCIAS}

BONACIM, Carlos A. G.; ARAUJO, Adriana M. P. Avaliação de desempenho econômico-financeiro dos serviços de saúde: os reflexos das políticas operacionais no setor hospitalar. Revista Ciência \& Saúde Coletiva, Rio de Janeiro, v. 16, supl. 1, p. 1055-69, 2011. Disponível em: http://www.scielo.br/pdf/csc/v16s1/a38v16s1. pdf. Acesso em: 15 jun. 2019.

BOTELHO, Louise L. R.; CUNHA, Cristiano C. A.; MACEDO, Marcelo. O método da revisão integrativa nos estudos organizacionais. Revista Gestão e Sociedade, Belo Horizonte, v. 5, n. 11, p. 121-36, maio/ago. 2011. Disponível em: https://www.gestaoesociedade. org/gestaoesociedade/article/view/1220/906. Acesso em: 20 abr. 2019.

CINTRA, Renato F.; VIEIRA, Saulo Fabiano A.; HALL, Rosemar José; FERNANDES, Cristiano R. A informação do setor de faturamento como suporte à tomada de decisão: um estudo de caso no Hospital Universitário da UFGD. Revista Ciência \& Saúde Coletiva, Rio de Janeiro, v. 18, n. 10, p. 3043-53, 2013. Disponível em: https:// www.scielosp.org/scielo.php?script=sci_arttext\&pid=S1413-81232013001800029. Acesso em: 9 jun. 2019.

CLAUDINO, Hellen G.; GOUVEIA, Eloise Maria de L.; SANTOS, Sérgio R.; LOPE, Maria Emília L. Auditoria em registros de enfermagem: revisão integrativa da literatura. Revista Enfermagem, Rio de Janeiro, v. 21, n. 3, p. 397-402, jul./set. 2013. Disponível em: https://www.e-publicacoes.uerj.br/index.php/enfermagemuerj/ article/view/7550/5450. Acesso em: 16 jun. 2019.

COSTA, Elenilde P. da S. R.; ARRAIS, Alessandra da R. Faturamento Hospitalar aplicado ao serviço de parto: modelo alternativo ao fee-for-service. Revista Acta Paulista de Enfermagem, São Paulo, v. 31, n. 2, p. 170-80, mar. 2018. Disponível em: http:// www.scielo.br/scielo.php?script=sci_arttext\&pid=S0103-21002018000200170. Acesso em: 11 jun. 2019.

DORNELLES, Treice S.; GASPARETTO, Valdirene. Gerenciamento de processos: estudo em uma organização hospitalar catarinense. Revista de Gestão em Sistemas de Saúde, São Paulo, v. 4, n. 2, p. 57-72, jul./dez. 2015. Disponível em: http://www. revistargss.org.br/ojs/index.php/rgss/article/view/159. Acesso em: 16 jun. 2019. GRIFFIN, Ricky W. Introdução à administração. São Paulo: Ática, 2007. 
GUEDES, Gisele G.; TREVISAN, Danilo D.; STANCATO, Kátia. Auditoria de prescrições de enfermagem de um hospital de ensino paulista: avaliação da qualidade da assistência. Revista Administração Saúde, São Paulo, v. 15, n. 69, p. 71-8, abr./jun. 2013. Disponível em: https://pesquisa.bvsalud.org/portal/resource/pt/lil-704508. Acesso em: 24 maio 2019.

GUERRER, Gabriela F. F.; CASTILHO, Valéria; LIMA, Antonio F. C. Processo de formação de contas em um hospital de ensino especializado em cardiologia e pneumonia. Revista Eletrônica de Enfermagem, Goiânia, v. 16, n. 3, p. 558-65, jul./ set. 2014. Disponível em: https://www.fen.ufg.br/revista/v16/n3/pdf/v16n3a09. pdf. Acesso em: 2 jun. 2019.

GUERRER, Gabriela F. F.; LIMA, Antonio F. C; CASTILHO, Valéria. Estudo da auditoria de contas em um hospital de ensino. Revista Brasileira de Enfermagem, Brasília, v. 68, n. 3, p. 414-20, maio/jun. 2015. Disponível em: http://www.scielo.br/pdf/ reben/v68n3/0034-7167-reben-68-03-0414.pdf. Acesso em: 11 jun. 2019.

IBAÑEZ, Nelson; BITTAR, Olímpio José N. V.; SÁ, Evelin N. de C.; YAMAMOTO, Edison K.; ALMEIDA, Márcia F.; CASTRO, Cláudio G. J. Organizações sociais de saúde: o modelo do Estado de São Paulo. Revista Ciência \& Saúde Coletiva, São Paulo, v. 6, n. 2, p. 391-404, jan. 2001. Disponível em: http://www.scielo.br/pdf/csc/v6n2/7010. pdf. Acesso em: 9 jun. 2019.

MONTANA, Patrick J.; CHARNOV, Bruce H. Administração. 3. ed. São Paulo: Saraiva, 2010.

OLIVEIRA, Djalma de P. R. Sistemas, organização \& métodos: uma abordagem gerencial. 18. ed. São Paulo: Atlas, 2009.

PEDROSA, Karilena K. A.; SOUZA, Marília F. G.; MONTEIRO, Akemi I. O enfermeiro e o registro de enfermagem em um hospital público de ensino. Revista Rene, Fortaleza, v. 12, n. 3, p. 568-73, jul./set. 2011. Disponível em: http://www.repositorio.ufc. br/bitstream/riufc/12489/1/2011_art_kkapedrosa.pdf. Acesso em: 11 jun. 2019.

PERTILLE, Fabiane; ASCARI, Rosana A.; OLIVEIRA, Maíra C. B. A importância dos registros de enfermagem no faturamento hospitalar. Revista de Enfermagem UFPE, Recife, v. 12, n. 6, p. 1717-26, jun. 2018. Disponível em: https://pesquisa.bvsalud.org/ portal/resource/pt/biblio-982180. Acesso em: 13 jun. 2019.

ROBBINS, Stephen P.; DECENZO, David A. Fundamentos de administração: conceitos essenciais e aplicações. 4. ed. São Paulo: Prentice Hall, 2012. 
SANTOS, Hellen G.; ANDRADE, Selma M.; BIROLIM, M. M. Informações sobre causas externas em internações hospitalares: conhecimento e opiniões de médicos auditores após uma intervenção. Revista Acta Scientiarum, Maringá, v. 34, n. 1, p. 738, jun. 2012. Disponível em: http://docs.bvsalud.org/biblioref/2016/08/1406/892361170-1-pb.pdf. Acesso em: 1ㅇ jun. 2019.

SENTONE, Andreza D. D. Análise dos indicadores da qualidade na elaboração da prescrição de enfermagem em uma unidade de internação de um hospital universitário público. 2005. 123 f. Dissertação (Mestrado em Enfermagem Fundamental) - Curso de Enfermagem, Escola de Enfermagem de Ribeirão Preto, Ribeirão Preto, SP, 2005. Disponível em: https://www.teses.usp.br/teses/ disponiveis/22/22132/tde-04052005-135414/pt-br.php. Acesso em: 24 mai. 2019.

SILVA, Larissa G. C.; HADDAD, Maria do Carmo Fernandes Lourenço; VITURI, Dagmar Willamowius; JODAS, Denise Albieri; OTRENTI, Eloá. Dez anos buscando a melhoria da qualidade do cuidado de enfermagem em um hospital universitário. Revista de Enfermagem e Atenção à Saúde, Uberaba, v. 1, n. 1, p. 44-56, 2012. Disponível em: http://seer.uftm.edu.br/revistaeletronica/index.php/enfer/article/view/328/284. Acesso em: 24 maio 2019.

SILVA, Marcia Z.; MORETTI, Bradlei R.; SCHUSTER, Herivelton A. Avaliação da eficiência hospitalar por meio da análise envoltória de dados. Revista de Gestão em Sistemas de Saúde, São Paulo, v. 5, n. 2, p. 100-15, jul./dez. 2016. Disponível em: http://www.revistargss.org.br/ojs/index.php/rgss/article/view/248. Acesso em: 26 maio 2019.

SOUZA, Marcela T.; SILVA, Michelly D.; CARVALHO, Rachel de. Revisão integrativa: o que é e como fazer. Revista Einstein, São Paulo, v.8, n.1, p. 102-106, 2010. Disponível em: http://www.scielo.br/pdf/eins/v8n1/pt_1679-4508-eins-8-1-0102. Acesso em 20 abr. 2019

VITURI, Dagmar W.; MATSUDA, Laura M. Os registros de enfermagem como indicadores da qualidade do cuidado: um estudo documental, descritivoexploratório e retrospectivo. Revista OnLine Brazilian Journal Nursing, Niterói, v. 7, n. 1, abr. 2008. Disponível em: https://pesquisa.bvsalud.org/portal/resource/ pt/lil-489725. Acesso em: 24 maio 2019. 